\title{
REVIEWS
}

GENETICS AND CANCER : Symposium on the Genetics of Cancer. Brit. J. Cancer, vol. 2, part 2, pp. 87-176.

Certain wide generalisations can be made about the causation of cancer. Thus there can be no doubt that one of the agents responsible for the change from the normal to the malignant state is long-continued irritation at a point. Yet such an environmental stimulus will initiate malignancy in some individuals but not in others. Furthermore, it does not do so with equal readiness in all parts of the body : for instance, the mucous membrane of the nose is much subject to irritation but it rarely becomes cancerous.

To make such statements as these is to propound problems in Genetics. Yet it is fair to say that of all aspects of cancer research the genetic has received least attention, and that medical practitioners find the results it has so far yielded neither readily accessible nor easily understood ; for no general survey of them is available, and one is much overdue. In order to supply that need, the Genetical Society of Great Britain and the British Empire Cancer Campaign sponsored a Symposium on the Genetics of Cancer, which was held in London on the 24th and 25th June 1948. Those responsible for the arrangements realised that it would not be possible to survey the whole field of the genetics of cancer in the time available. They therefore decided to limit the discussion to three of its most important aspects : The inheritance of cancer in animals, Virus and carcinogen induced mutations, and The inheritance of cancer in Man. In all, twenty-one papers were delivered, and these are published in the British Fournal of Cancer for 1948.

The importance of the genetic aspect of cancer, and of the cancer phenomena in biology, becomes apparent on reading these abstracts, as it did during the course of the Symposium. The field which they cover may more usefully be reviewed by a survey of certain principles which underlie them, and of some conclusions to which they lead, than by a mere summary of their contents.

The hereditary material is transmitted in the form of self-perpetuating units, the genes, which are carried both in the nucleus and in the cytoplasm. As Darlington points out in his contribution, a graded series may be traced from those genes, the plastids and others, which exist only in the cytoplasm, through the proviruses to the true virus particles which can infect the cytoplasm and there perpetuate themselves, such as those responsible for infective warts. The essential feature of all the genes is that they do not contaminate one another or "blend," so that from this aspect at least they retain their identity instead of producing a chaotic mixture. Furthermore, those carried in the nucleus possess an additional property of fundamental importance. Owing to the existence of the chromosome mechanism and the operation of bisexual reproduction, even those nuclear genes which have arisen far apart in time or space may ultimately be brought together, and in an immense variety of combinations some of which may be beneficial to the individual in which they occur. This outstanding advantage, conferring as it does great genetic variability, is absent from the genes of the cytoplasm, so it is not surprising to find that the hereditary material is consigned principally to the nucleus. Clearly it necessitates a further property in addition to those so far mentioned, that the genes should have a high degree of permanence. That is to say, they should be intrinsically very stable or, to use a different terminology, they must seldom mutate. For high mutation-rates would imperil any new advantageous combination as surely as would genic blending. Moreover, changes in the structure of the genes are not related 
to the needs of the individual ; consequently they will usually be disadvantageous since it is far easier to damage, than to improve, a highly organised system, such as that presented by the body of even the most primitive animals. Consequently the cytoplasmic, as well as the nuclear, genes should not be highly mutable, otherwise those best adjusted to the organism would not be sufficiently permanent.

Thus mutation-rates should be in equilibrium between the need to provide genetic diversity and the need to preserve certain genic combinations. The optimum mutation-rate will vary for different genes and must be reached, or approached, by selection. Consequently, it appears to be a necessity, as well as a physiological probability, that genes should exist which affect mutation. This indeed is now established, for it has been possible to modify the mutation rate in Drosophila by selection; indeed in this Symposium, Demerec describesa single gene controlling mutation, its presence being necessary to make the individual react to mutagenic substances. However, it may well be argued that selection has achieved genic stability only in so far as is compatible with the frequency required and the large size of the molecules concerned, and that the true optimum mutation-rates may often be lower than those which it has been possible to obtain.

What has been said of the necessarily disadvantageous nature of most mutations must apply also to any variations occurring at random. For the bodily (as the mental) characteristics of organisms are due to the action of genes operating in given environments ; alter either the genes, by mutation or recombinations, or else the environment, and the result may be changed and variation ensue. The normal individual of any successful species must clearly possess a genic equipment which reacts harmoniously with the environmental range to which it is usually exposed. This necessitates a delicate adjustment built up and maintained by selection. So that not only mutations, but recombinations and environmental experiences beyond the ordinary range, will generally have harmful consequences. Thus though an immense fund of genetic variability is essential for evolutionary change, the occurrence of physiological maladjustments will be opposed by selection within the limits which it can control, whether due to mutation, recombination, or the environment (for a wide range of adaptations is concerned with escaping deleterious environmental effects).

Now selection is powerless to control harmful qualities arising beyond the normal span of life. It may also be ineffective in adjusting the population to overcome very rare defects; not because there is any minimum value for the amount of variability which selection can influence, but because the process of adjustment in respect of very rare variation may be so slow that what is achieved in the earlier stages may become inappropriate in the later ones owing to other and more rapid evolutionary changes. Moreover, the frequency of very rare conditions may approach their mutation-rates and, as already suggested, these cannot be lowered indefinitely.

There is some reason therefore to suspect that malignant degeneration of epithelial cells is sufficiently common for selection to have buffered the body against it with great efficiency. Carcinomas are really very rare until early middle age. In the conditions of primitive societies, and earlier, when the adjustments must have taken place, very few individuals would reach the age of forty, and the population would virtually be free from such cancers. They only constitute a problem beyond the usual age of reproduction and beyond the normal span of life except when prolonged by the conditions of civilisation. Even xeroderma pigmentosum is no exception, since this is a unifactorial condition which has been pressed into almost complete recessiveness.

In these respects, the connective tissue, and related cells, are in sharp contrast ; for the sarcomas occur almost as often in youth as in age. They are at least as frequent as carcinomas in the young, though they become relatively so uncommon in later life. They indeed seem to constitute a rare disease against which it has not been possible to protect the body even in adolescence. 
Thus it is likely that the considerable freedom from carcinomas enjoyed until early middle age is an achievement of evolution and one which has occupied a long period of time. If so, we can scarcely hope to extend it into later life.

A given type of defect can usually be produced by various means, for each feature of the adult body is the product of a long chain of reactions which may be interfered with at many points in such a way as to inhibit, or else to exaggerate, the result. Thus the normal red eyes of Drosophila melanogaster can be changed genetically to white in more than one way (due to the definitive "white-eye" gene at locus I, I.5, or to the interaction of the genes responsible for brown and for scarlet eye colours). In man, at least five distinct genes, two in all probability allelomorphs, each give rise to retinitis pigmentosa while, to take another example also from man, diabetes insipidus can be produced by a gene recessive in effect or else it may supervene as the result of destructive changes at the base of the pituitary. In fact it is a commonplace of genetics to find distinct genes, or genic combinations, with apparently identical effects on some or all of the characters they control; so too, environmental changes simulating the action of known genes are frequently encountered.

These considerations apply to the production of cancer as well as to any other condition, and indicate how dangerous it is to generalise too widely on the subject. Thus we find that the same type of cancer may be under different genetic control, and require a different environmental stimulus for its realisation, in distinct species even when closely related. Genetic studies of cancer have not been conducted on any species at all closely related to Man, so that the results of such work, while of great importance for the general theory of the disease, are not applicable in detail to the human situation. That perhaps is self-evident, but the point introduces certain complexities as it is pressed further. No one would expect the causation of very distinct types of cancer, carcinomas and sarcomas for example, to be identical in Man ; but the general point of view raised here would lead us to expect that genes which cause greater liability to cancer in one part of the body do not necessarily raise the susceptibility elsewhere, and this is what we find. The data of Penrose given in the Symposium indicate that female relations of those who suffer from cancer of the breast show an unduly high incidence of that form of the disease, but not of cancer in other situations. Smithers in his article agrees with this result, and has obtained no evidence that breast cancers arise at an earlier age where there is a family history of them. The extensive data of the Danish Cancer Registry, which are discussed by Tage Kemp, also demonstrate the existence of hereditary susceptibility to breast (and to other) cancers but, contrary to the findings of Penrose and Smithers, they indicate that a family history of breast cancers is associated with earlier age of onset and with a somewhat greater tendency to cancer in other parts of the body. Evidently these results are not necessarily discordant : the situation may not be quite the same in the English and Danish populations.

The papers read at the Symposium on the Genetics of Cancer show that every gradation exists in the genetic control of that disease. At one end of the series is the tendency, determined on a multifactorial basis, to raise slightly the susceptibility of the body as a whole to malignant change. At the other extreme are unifactorial conditions interacting with unescapable environmental stimuli to produce cancer with a certitude almost comparable to segregation in a simple Mendelian experiment : xeroderma pigmentosum is one out of several instances of the kind. Yet it is relevant to the point of view developed here that Koller now reports a mild form of that condition which may well be due to another allelomorph of the gene responsible for the well-known, though rare, severe form.

Evidently large numbers of genes may, with varying degree and with different environmental stimuli, be carcinogenic. Consequently, recombinations, and mutations of both nuclear and cytoplasmic genes, must be agents in producing cancer. This is indeed indicated by the important discovery of Demerec, who shows that some carcinogenic substances also produce mutations and that others 
do not : a result which strongly suggests the diverse nature of the cancer-producing processes.

Those who read the abstracts of this Symposium can hardly doubt that hereditary tendencies play a part in the causation of cancer. However, the considerations just outlined suggest that while it is possible frequently to generalise from the genetic situation found in one pedigree to that expected in another, caution should be exercised in doing so. Nevertheless, the knowledge that such hereditary tendencies exist is likely to be of use to practitioners. Advice in regard to marriage can clearly be given to members of those families in which pre-cancerous states are inherited on a simple basis (such as xeroderma pigmentosum, a recessive with slight heterozygous manifestation, or pre-cancerous rectal polypi when due to a gene effective in the heterozygote). Such conditions are rare, but the facts of genetics suggest a caution of wider application : that the possibility of cancer should particularly be kept in mind when treating members of a family in which several cases of the disease have occurred, such as those illustrated in the pedigrees communicated to the Symposium by Smithers and Tage Kemp. Within the last five years I have myself encountered two relevant instances of this kind. In both of them the patient experienced for some time vague abdominal discomfort with occasional pain for which he had received palliative treatment, and an eventual exploratory operation demonstrated a cancer which by then had become inoperable. These two men each had a family history of the disease, which had occurred in the mother and brother of one and in a brother, a sister and an aunt of the other. Had this fact been taken into account, it is possible that suspicion might have been aroused earlier, perhaps in time for the removal of the growth to be attempted with some hope of success.

All will agree that early diagnosis is the pre-eminent requisite in the treatment of cancer, moreover the first step towards securing it is almost entirely in the hands of the patient. If it were generally appreciated that the probability of cancer is rather higher in some families than in others, those with relations who have developed the disease might pay attention earlier to suspicious symptoms when arising in middle or later life.

There are certain to be some who feel that the public ought not to be told that any such hereditary susceptibility exists, holding that much unnecessary alarm would be given to the relatives of cancer patients. I do not share that view, believing rather that what is true had better be known. The essential co-operation of patients may best be secured by making clear the relevant facts relating to cancer, and its genetic aspect could play some part in securing its early recognition, upon which all hope of cure depends.

E. B. FORD.

\section{THE MOSCOW CONFERENCE ON GENETICS}

\section{SOVIET BIOLOGY. By T. D. Lysenko. A report to the Lenin Academy of Agricultural Sciences. Moscow 1948. Translated for the Science Section of the Society for Cultural} Relations with the U.S.S.R. Pp. 51. London : Birch Books. 2s. 6d.

This address by Academician T. D. Lysenko to the Lenin Academy of Agricultural Sciences reports results so remarkable that they raise questions beyond the scope of an ordinary review. Most writers on biology, especially when dealing with their own work, freely and clearly give all relevant details of their experiments so that the reader can decide whether or not the results and conclusions the author arrives at are justified. It is regrettable that so often in this book and in earlier publications by the author and his associates, such details are not adequately given. We have, however, heard so much about the work of Lysenko and Michurin that all biologistsand especially those engaged in growing, grafting and breeding plants-will, I feel, be impelled to read this latest account of biology in the Soviet Union. There are 